\title{
Urachal Pathology: Review of Cases
}

\author{
Lorena García García Begoña Ballesta Jonathan Rodríguez Talavera \\ Nuria Morales Orribo Almudena Carrión \\ Department of Urology, Hospital Universitario Nuestra Señora de Candelaria, Santa Cruz de Tenerife, Spain
}

\section{Keywords \\ Urachus · Umbilical abscess · Umbilical sinus}

\begin{abstract}
Introduction: The urachus is an embryologic remnant which is formed from the obliteration of the allantois. Urachal abnormalities are caused when defective obliteration of the urachus happens. They are an infrequent condition. Incidence is estimated to be between 5,000 and 8,000 live births. Its diagnosis and management remain a challenge due to the lack of an specific clinical picture and the controversy about the management. Objective: The objective of this study is to assess the clinical presentation, diagnosis, therapeutic management, and outcomes of urachal anomalies in our health area. Materials and Methods: We performed a retrospective review of all cases of urachal anomalies recorded Tenerife (southern health area), La Gomera, and El Hierro Islands during a 5 year period. Results: Twenty-three cases of urachal pathology were included. The mean age of presentation was 32 years old. $73.9 \%$ were male. $65 \%$ were diagnosed in adults. In $30.3 \%$ of the cases, it was a casual finding. Symptoms included fever, umbilical exudate, hematuria, abdominal pain, and umbilical granuloma. The main diagnostic tests were ultrasound and computed tomography scan. Treatments were selected conservative management $(43.5 \%)$, selective resection, partial cystectomy, and radical cystectomy. All patients had a good evolution. However, 2 cases where benign tumors were suspected, clinically, had a final histology of cancer in the specimen. Conclu-
\end{abstract}

karger@karger.com

(c) 2021 S. Karger AG, Basel

www.karger.com/uin sions: Due to the lack of a specific clinical picture and undefined findings in image tools, diagnosis is difficult and it may be inaccurate. Despite more data are needed, our results suggest that the systematic excision of urachal lesions could result in safer outcomes since cases where a benign lesion is clinically suspected might result in malignant tumors.

(c) 2021 S. Karger AG, Basel

\section{Introduction}

The urachus or median umbilical ligament is an allantois remnant of embryologic bladder descent into the pelvis found between the umbilicus and the dome of the bladder. Under normal conditions, this structure is a thin fibrous cord [1]. Usually, the urachus involutes in uterus or early childhood forming the median umbilical ligament. However, it may persist and give rise to a spectrum of pathological anomalies [2].

The pathologies associated with urachus may be congenital or acquired. Congenital urachal pathologies include patent urachus (48\%), umbilical urachal sinus (18\%), and vesico-urachal diverticulum (3\%). Acquired urachal pathologies include repermeabilization, infection, and, although urachal tumors constitute $<1 \%$ of all bladder tumors, malignancy [3].

The incidence of urachal pathologies in childhood is approximately 1 per 5,000-8,000 newborns. Male to female ratio is $2: 1$. In adults, it is rare because urachal anomalies usually involute in early childhood $[4,5]$. The 


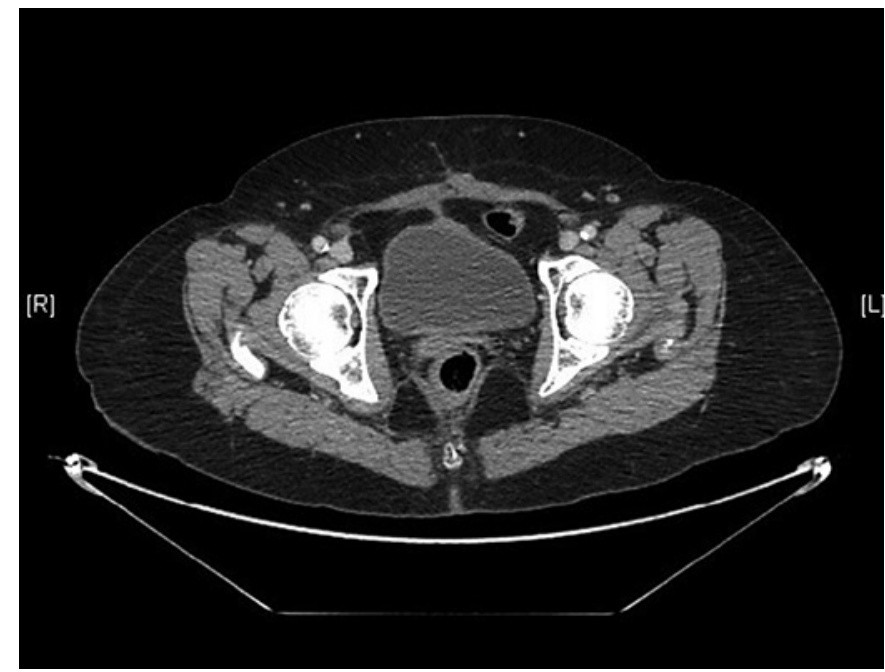

Fig. 1. Patent urachus valued by CT. CT, computed tomography.

adequate and early diagnosis of urachal pathologies is very important. Adults have a higher risk of urachal cancer with an estimated incidence of 0.18 per 100,000 .

Clinically, there may show umbilical or urinary discharge, umbilical mass, vague abdominal pain, or hematuria. Urine discharge from umbilicus suggests patent urachus. Hematuria points to vesico-urachal diverticulum. The presence of pus discharged from umbilicus may be present in urachal sinus. Further complications such as infection and malignancy may occur.

The presentation and progression of the disease in pediatric and adult population are different. Its diagnosis remains a challenge due to the lack of an specific clinical picture.

Ultrasound (US) is often the first test performed in patients with suspected urachal cysts. Computed tomography (CT) is a good confirmation tool when US leads to ambiguous findings. Cystography, magnetic resonance urography, and sino/fistulography are also useful in specific cases. Sometimes the final diagnosis is made with surgical exploration $[1,6]$. Regarding management, there is controversy on whether prophylactic surgery should be performed in all patients with urachal anomalies (Fig. 1,2).

\section{Methods}

We made a retrospective review of all patients diagnosed with any kind of urachal remnant between 2006 and 2020 in Nuestra Señora de Candelaria University Hospital in Tenerife, Spain, which includes all cases from Tenerife (southern health area), La Gomera, and El Hierro Islands throughout the time period. We

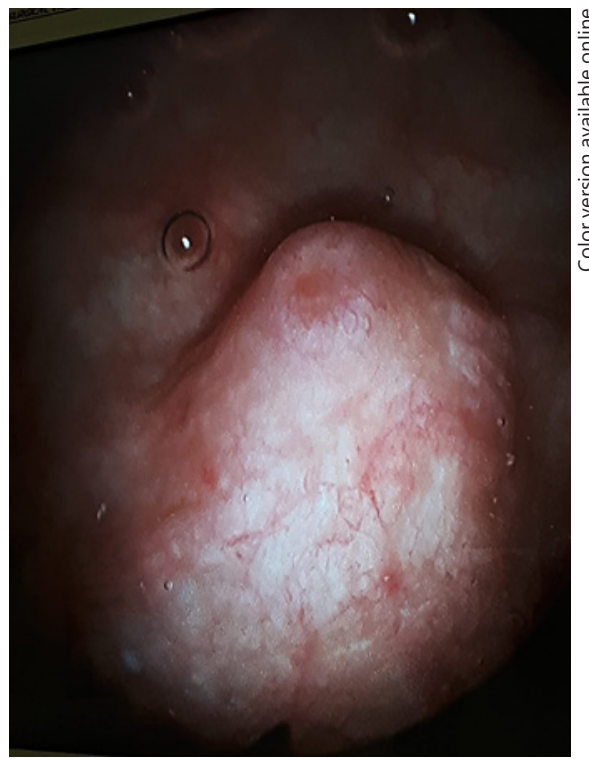

Fig. 2. Endoscopic vision of an urachal cyst.

recorded age, gender, presenting symptoms, diagnostic tools, type of urachal remnant, management, histology follow-up schemes, and short- and long-term complications after treatment.

\section{Results}

Twenty-three cases of urachal pathology were included. The mean age of presentation was 32 years old (0-78). $73.9 \%(17 / 23)$ were men. $26.1 \%(6 / 23)$ were females. $65 \%$ $(15 / 23)$ were diagnosed in adults (older than 18 years).

In $30.3 \%(7 / 23)$ of the cases, it was a casual finding. Fever was recorded in $30.3 \%$ (7/23) of the patients, $26 \%$ $(6 / 23)$ had umbilical exudate, $17.4 \%(4 / 23)$ showed hematuria, and $13 \%(3 / 23)$ had abdominal pain. One out of 23 presented with umbilical granuloma.

The main diagnostic tests used were abdominal US and CT scan used in $43 \%$ of the cases each. In 2 cases, the 2 tests were combined. US + cystography, US + magnetic resonance imaging, CT + cystoscopy, and fistulography were also chose as the diagnostic tools within this series (see Table 1).

Most common clinical diagnoses were urachal cyst (60.9\%). Adenocarcinoma was only clinically suspected in 1 case $(0.04 \%)$, and there were doubts with a differential diagnosis with urachal diverticulum.

Regarding the therapeutic approach, conservative treatment was selected in $43.5 \%(10 / 23)$ of the cases. In contrast, surgery was performed in $56.5 \%$. In 9 patients, a selective resection of the urachal anomaly was carried 
Table 1. Descriptive data of the study

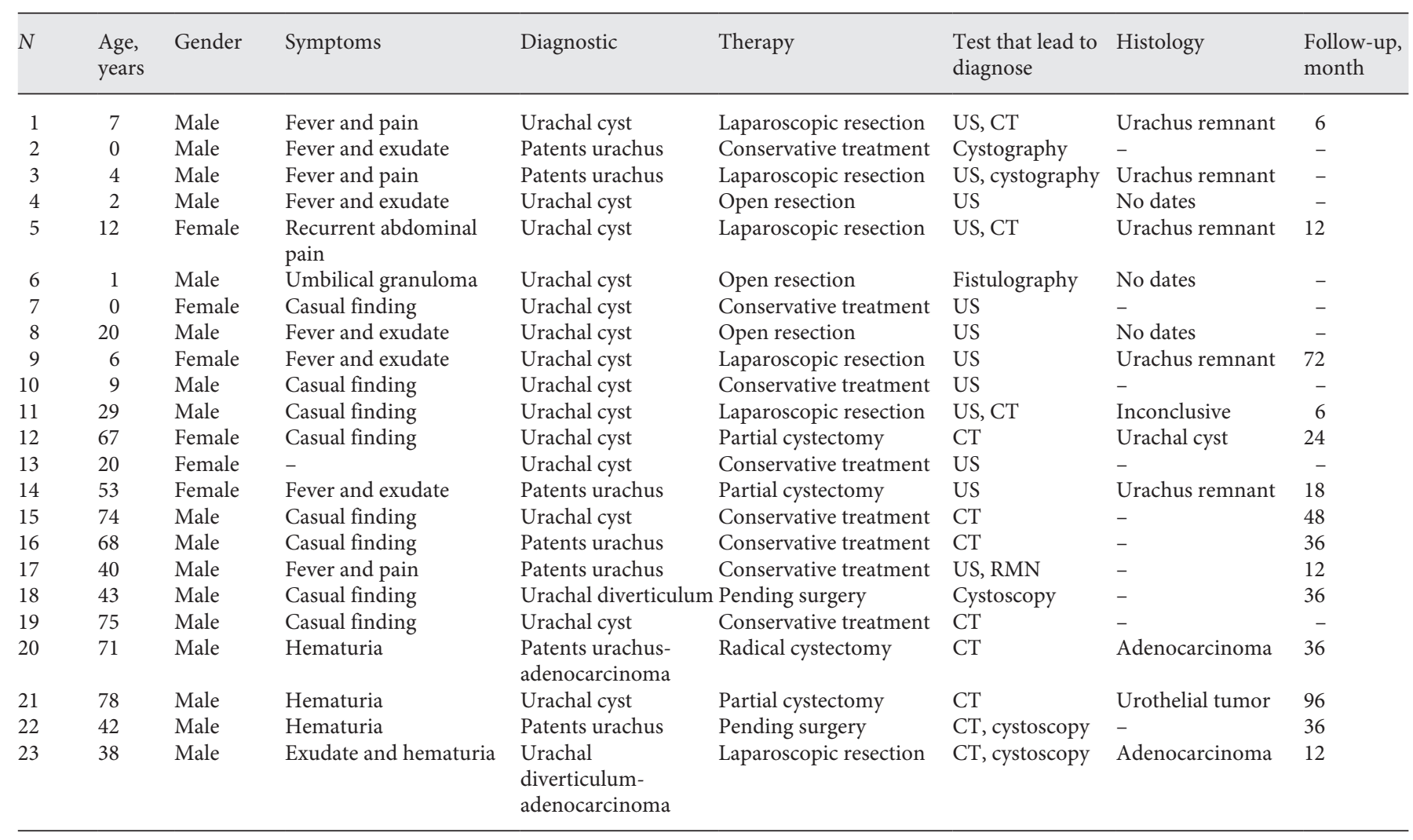

CT, computed tomography.

out. Approach was laparoscopic 6 versus open in 3.5 out of 6 of the cases in the group of laparoscopic resection have a final histology of urachus remnant. However, there was 1 case where no malignancy was suspected in the clinical diagnosis and final histology resulted in adenocarcinoma (case \#20). Partial cystectomy was chosen in 3 cases and radical cystectomy in 1 . Between them, 2 were benign tumors and the rest were urothelial carcinoma and adenocarcinoma. The prior clinical diagnosis for the case of the urothelial carcinoma was urachal cyst (case \#21).

Long-term follow-up was only found in 14 cases. Within them, the mean time period was 32 months (range 0-96). Nor short- nor long-term complications were registered for any case whatsoever. No recurrence was recorded either.

\section{Discussion}

Several authors suggest that systematic excision of urachal lesions which are detected during childhood should be performed in order to prevent infection or other prob- lems in adulthood as the development of malignancy in residual remnants $[2,5,7,8]$, whereas others consider that conservative management, even in cases of infected anomalies $[9,10]$, results in better outcomes. For instance, Copp et al. [11] state that surgery should only be performed in cases with unfavorable histology that is suggestive of malignancy, that is, cases with transitional epithelium, squamous metaplasia, intestinal metaplasia, or mixed metaplasia.

In our series of urachal pathology, on the one hand, no bad outcomes were recorded in cases where conservative treatment was chosen with a top follow-up length of 48 months between those patients. However, on the other hand, despite the suspected clinical diagnosis was confirmed histologically in the majority of cases with $100 \%$ concordance, we registered 2 patients out of 23 (0.09\%) (cases \#20 and \#21) where no malignancy was suspected clinically but postsurgical histology resulted in cancer. Although we did not record histology in 13 cases out of the total, and data collected prospectively might enlighten more accurate conclusions, these find- 
ings should be taken into account. They might suggest that systematic excision of urachal lesions could result in safer outcomes.

\section{Conclusions}

Due to the lack of a specific clinical picture and undefined findings in image tools, diagnosis is difficult and it may be inaccurate. Despite more data are needed, our results suggest that the systematic excision of urachal lesions could result in safer outcomes since cases where a benign lesion is clinically suspected might result in malignant tumors.

\section{Statement of Ethics}

The CEIC Hospital Universitario Nuestra Señora de Candelaria considers that the urachal pathology review study respects basic ethical principles and complies with applicable legislation.

\section{Conflict of Interest Statement}

There are no conflicts of interest.

\section{Funding Sources}

There are no funding sources.

\section{Author Contributions}

All the authors of the review have contributed to the conception and design of the work. Dr. García, Dr. Ballesta, Dr. Carrion, and Dr. Orribo carried out the data acquisition and analysis. The writing and critical review were the work of Dr. García, Dr. Ballesta, and Dr. Talavera. The interpretation of the results and the final approval of the version were carried out jointly.

\section{References}

1 Hassan S, Koshy J, Sidlow R, Leader H, Horowitz M. To excise or not to excise infected urachal cysts: a case report and review of the literatura. J Pediatr Surg Cae Rep. 2017;22: $35 \mathrm{e} 38$.

2 Nogueras-Ocana M, Rodriguez-Belmonte R, Uberos-Fernandez J, Jimenez-Pacheco A, Merino-Salas S, Zuluaga-Gomez A. Urachal anomalies in children: surgical or conservative treatment? J Pediatr Urol. 2014;10:522e6.

3 Qureshi K, Maskell D, McMillan C, Wijewardena C. An infected urachal cyst presenting as an acute abdomen: a case report. Int J Surg Case Rep. 2013;4(7):633-5.

4 Yiee JH, Garcia N, Baker LA, Barber R, Snodgrass WT, Wilcox DT. A diagnostic algorithm for urachal anomalies. J Pediatr Urol. 2007; 3(6):500-4.
5 Mistry KA, Khatri GD, Sood D, Sharma S, Morey P, Sood S, et al. Late presentation of congenital urachal sinus in a middle aged male complicated by an umbilical abscess: a case report. Egypt J Radiol Nucl Med. 2015; 46(3):755-9.

6 Cilento BG, Bauer SB, Retik AB, Peters CA, Atala A. Urachal anomalies: defining the best diagnostic modality. Urology. 1998;52(1): 120.

7 Donate Moreno MJ, Jimenez Bachs JM, Salinas Sanchez AS, Lorenzo Romero G, Segura Martín M, Hernández Millán I, et al. Patología del Uraco: revisión de conjunto y presentación de tres casos. Actas Urol Esp. 2005; 29:332e6.
8 Gimeno Argente V, Domínguez Hinarejos C, Serrano Durbá A, Estornell Moragues F, Martínez Verduch M, García Ibarra F. Quiste de uraco infectado en edad infantil. Actas Urol Esp. 2006;30(10):1034e7.

9 Ueno T, Hashimoto H, Yokoyama H, Ito M, Kouda K, Kanamaru H. Urachal anomalies: ultrasonography and management. J Pediatr Surg. 2003;38(8):1203.

10 Lipskar AM, Glick RD, Rosen NG, Layliev J, Hong AR, Dolgin SE, et al. Nonoperative management of symptomatic urachal anomalies. J Pediatr Surg. 2010;45(5):1016.

11 Copp HL, Wong IY, Krishnan C, Malhotra S, Kennedy WA. Clinical presentation and urachal remnant pathology: implications for treatment. J Urol. 2009;182(4 Suppl 1):1921. 